\title{
Study of the lines of common wheat of breeding of National Center of Grain named after P.P. Lukyanenko on allele variants of Waxy-genes
}

\author{
Davoyan E.R.*, Davoyan R.O., Zubanova Y.S., Mikov D.S., Boldakov D.M., \\ Bespalova L.A., Agaeva E.V., Bukreeva G.I. \\ National Center of Grain named after P.P. Lukyanenko, Krasnodar, Russia \\ *e-mail:davayan@rambler.ru
}

The aim of this work was the molecular identification of promising genotypes common wheat by allele variants of Waxy genes $(W x)$ to create varieties with improved technological qualities of grain. 352 lines of common wheat were studied by allelic variants of genes $W x A 1, W x B 1, W x D 1$. All lines were obtained in the department of breeding and seed production of wheat and triticale in the National Center of Grain named after P.P. Lukyanenko, by crossing with a mutant line carrying null alleles $W x A 1 b, W x B 1 b$ and $W x D 1 b$. There were identified 205 lines carrying the wild type allele $W x A 1,229$ lines with the wild-type allele of the gene $W x B 1$ and 249 with the wild-type allele of the gene $W x D 1.64$ lines carried functional allele $W x B 1 e$, different from that wild type. The lines carrying zero alleles of genes $W x A 1, W x B 1$ were selected (147 and 58 respectively). 13 lines, showing the heterozygous condition for the gene $W x D 1$, and one line for the $W x B 1$ gene, were identified. Full technological evaluation was given to 57 promising lines and several standard varieties. The obtained results show that the lines carrying zero-allele $W x A 1 b$ or $W x B 1 b$, as well as a combination of alleles $W x A 1 b+W x B 1 b$, are generally characterized by high protein content (15.3, $15.4,15.5 \%$ respectively), gluten $(28.3,28.6,30.3 \%)$. All three groups of lines are close to the "strong" wheat by the strength of flour (285 alveograph units, 270 a.u., 279 a.u.). The lines with the identified null alleles of the $W x$ genes are distinguished by a high water absorption capacity compared to the Tanya variety, which does not have these alleles in its genotype. The optimal combination of liquefaction indicators and high valorimetric evaluation probably contributes to the formation of high-volume bread with a high overall baking rating. 